\title{
SUCCESS-DRIVING BUSINESS MODEL CHARACTERISTICS OF IAAS AND PAAS PROVIDERS
}

\author{
Sebastian Floerecke and Franz Lehner \\ Chair of Information Systems (Information and IT Service Management), \\ University of Passau, Passau, Germany
}

\begin{abstract}
Market analyses show that some cloud providers are significantly more successful than others. The research on the success-driving business model characteristics of cloud providers and thus, the reasons for this performance discrepancy is, however, still limited. Whereas cloud business models have mostly been examined comprehensively, independently from the distinctly different cloud ecosystem roles, this paper takes a perspective shift from an overall towards a selective, role-specific and thereby ecosystemic perspective on cloud business models. The goal of this paper is specifically to identify the success-driving business model characteristics of the so far widely neglected cloud ecosystem's core roles, IaaS and PaaS provider, by conducting an exploratory multiple-case study. 21 expert interviews with representatives from 17 cloud providers serve as central data collection instrument. The result is a catalogue of generic as well as cloud-specific, subdivided into role-overarching and role-specific, business model characteristics. This catalogue supports cloud providers in the initial design, comparison and revision of their business models. Researchers obtain a promising starting and reference point for future analysis of business models of various cloud ecosystem roles.
\end{abstract}

\section{KEYWORDS}

Infrastructure as a Service (IaaS), Platform as a Service (PaaS), Success-Driving Business Model Characteristics, Cloud Computing Ecosystem, Exploratory Multiple-Case Study.

\section{INTRODUCTION}

Several cloud providers encounter major difficulties to design and implement suitable business models. This is the reason why many are still experimenting with a variety of business models aiming to establish a sustainable and profitable position within the cloud ecosystem $[1,2]$. Market analyses (e.g., [3,4]) show that some cloud providers are significantly more successful than others in this respect. Whereas, for instance, Amazon Web Services is the leading provider of Infrastructure as a Service (IaaS), controlling about 52 percent of the sector [5], others, such as Cisco, Dell and Hewlett Packard Enterprise, are examples for providers that already shut down their own public IaaS offering. The research on the success-driving business model characteristics of cloud providers and thus, the reasons for this performance discrepancy is, however, still limited [6-8].

So far, research has only provided one extensive analysis of cloud business models and their connection to economic success: Labes et al. [6] derived abstract success factors by relating publicly available information regarding predefined characteristics of business model components of selected providers to their web visibility and profit. However, the study neglected that the cloud ecosystem allows the adoption of various roles and thus, cloud providers are characterized by a high degree of heterogeneity [9]. Beyond this background, this paper takes a perspective shift from an overall perspective towards a selective, role-specific and thus ecosystemic perspective on cloud business models. The core of the cloud ecosystem consists of providers of the three basic, interrelated and consecutive cloud service layers - Infrastructure as a Service (IaaS), Platform as a Service (PaaS) and Software as a Service (SaaS) $[10,11]$. These three roles form 
the heart of the whole cloud business and are forecasted to account for about 70 percent of the overall turnover of the cloud ecosystem in 2019 [12].

Whereas several, largely isolated contributions have examined SaaS provides [8, 13-16] from a success perspective, IaaS and PaaS providers have been widely ignored [17]. This paper attempts to fill this gap and therefore addresses the following research question: What are the business model characteristics influencing the success of the cloud ecosystem's core roles IaaS and PaaS provider?

To answer this research question, an exploratory multiple-case study [18] is conducted. Case studies have proven their effectiveness in identifying success factors (e.g., [19, 20]). 21 expert interviews with representatives from 17 cloud providers serve as central data collection method. The success-driving business model characteristics are, in contrast to Labes et al. [6], collected impartially, this means without preselection, directly from the interviewed cloud experts.

The rest of this paper is organized as follows: Section two contains the related work regarding cloud computing, business models, business ecosystems and success factors. In the third section, the conducted case study research approach is depicted in detail. The identified success-driving business model characteristics are listed and described separately according to the various business model components in section four. In section five, the results are discussed and several unexpected aspects concerning the current situation and possible future developments within the cloud ecosystem are presented. Section six provides a summary, limitations, contributions for theory and practice and a prospect on future research.

\section{RELATED WORK}

Cloud computing is a relatively new IT operations model, which has radically transformed the way IT resources are produced, provided and used [21]. This is the reason why numerous scholars (e.g., $[10,22])$ consider cloud computing as a co-evolution of computing technology and business models. The main characteristics of cloud services, including on-demand self-service, broad network access, resource pooling, rapid elasticity and measured service, distinguish it from its traditional counterpart, the on-premise IT [23]. Cloud services can be delivered via four different deployment models: public, private, hybrid and community. These deployment models particularly differ in their degree of operational isolation, the access control to a specific cloud service and the physical location of the underlying hardware servers [24].

With the introduction of cloud computing by Amazon Web Services in 2006, both vendors of traditional IT services and start-up companies were given the opportunity to take up new roles in this emerging complex and network-like business ecosystem [9, 10]. A role in this context is understood as a "[...] set of similar services offered by market players to similar customers" [22]. A business ecosystem represents a pertinent scope for systemic innovations, where different interrelated and interdependent companies cooperate to deliver full-scale customer solutions [25]. Compared to traditional linear and customer-focused value chains in IT service provision, the value creation is not an exclusively linear process with upstream and downstream actors anymore. Value is, instead, created in a network of companies with many both vertical and horizontal relations [26]. The cloud ecosystem's core consists of providers of the three basic service layers IaaS, PaaS and SaaS [10, 11]. IaaS supplies infrastructural resources (compute, storage and network). PaaS allows users to develop and deploy applications by offering a software development environment with programming languages, libraries and tools. SaaS refers to providing ondemand applications [23, 24]. Building upon these three consecutive and interrelated service layers, a multitude of further ecosystem roles, such as aggregators, integrators and market place operators, has evolved [9]. 
From a business model perspective, a business ecosystem consists of synergetic business models, which enable joint value creation and capture [27]. Companies within a business ecosystem are thus interconnected through their business models [28]. This circumstance emphasizes the position of a company (its role) within a business ecosystem as the primary driver and impact factor for the design of its business model(s) [29]. As each ecosystem role is related to specific business opportunities, it also demands a different business model [30]. Nonetheless, companies fulfilling the same ecosystem role still have a wide range of business model design options to distinguish from one another. In conclusion, a business model can be defined as a detailed specification of how ecosystem roles are realized by individual companies [31, 32]. As each occupied ecosystem role has to be implemented in the form of (at least) one business model, a firm can employ several business models simultaneously [27, 33]. Designing and running such a business model portfolio is challenging, however, it offers the great potential to exploit synergies, for instance, by reusing capabilities and resources, across business models [34].

Apart from the ecosystem lens, a business model is, in general, a system comprising a set of interrelated components or partial models for depicting, implementing and evaluating the business logic of a company $[35,36]$. It builds upon central theories, in particular, the resource-based view, the transaction cost theory, the cooperation theory and the strategic network theory [29]. According to Amit and Zott [37], such a cross-theoretical perspective is necessary as no existing single theory can fully explain value creation. Business models are mostly classified as an intermediary between a company's strategy and its business processes. Accordingly, a business model is the conceptual and architectural implementation of a business strategy and, in turn, represents the foundation for the realization of business processes [38]. Commonly, business models are considered as static, even though they are subject to external factors, such as competitive forces, social or technological change, varying customer opinions and the dynamic political and legal environment [39]. The literature provides a multitude of cross-industry and industry-specific business model frameworks, which include design options for various subsets of components $[31,36]$. A comprehensive and widespread cross-industry framework among both practitioners and scholars is the Business Model Canvas [40]. This framework comprises the following nine components: value propositions, key resources, key activities, partner network, customer segments, channels, customer relationships, revenue streams and cost structure.

Figure 1 summarizes the connection between a cloud provider, his occupied ecosystem roles, their instantiated business models, their components and their multiple design options for component-specific business model characteristics:

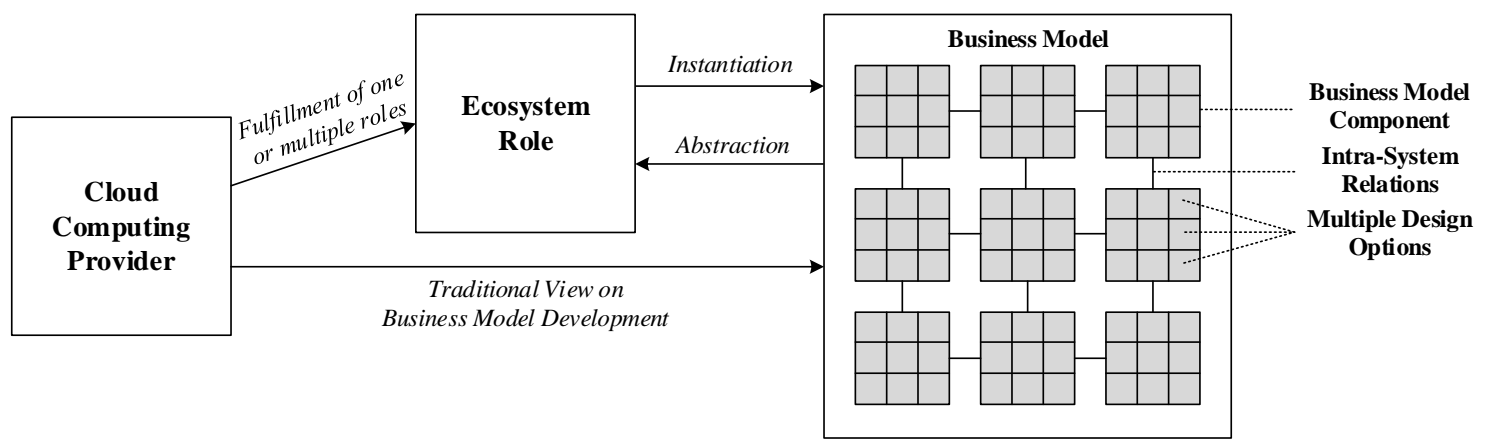

Figure 1. The Connection between Cloud Providers, Ecosystem Roles and Business Models

Overall, the research on cloud business models is nascent: Giessmann and Stanoevska-Slabeva [41] proposed a classification model for PaaS providers' business models and hypotheses regarding future developments. Giessmann and Legner [42] published a set of possible design principles guiding software providers to define PaaS business models (without reference to success). 
Based on the available market offerings, Naous et al. [43] designed a classification scheme for Analytics as a Service (AaaS) business model configurations and derived five business model archetypes. A further specialized area is the cloud-based continuous auditing, for which Kiesow and Thomas [44] created a first draft of a business model. The only holistic cloud-specific business model framework was developed by Labes et al. [33]: their morphological box entails various categories representing the basic components of a business model, each broken down into design features that show possible design options [33]. Based on this, Labes et al. [6] analysed the business models of selected cloud providers, matched them with the framework and identified four common patterns of cloud business models. Apart from that, researchers examined the fundamental impacts of the shift from delivering on-premise IT applications to cloud services with regard to business models (e.g., $[1,2,45])$. In addition, scholars have dealt with the process of transforming an on-premise business model to a cloud business model (e.g., [46-48]). Ebel et al. [49] built and evaluated a software tool to support the business model creation. Another research strand aims to explore the individual aspects within cloud business models, which may have a positive influence on the growth of the general adoption level of cloud services compared to the on-premise IT among customers (e.g., [50, 51]). A literature study of Labes et al. [52] shows that several further contributions have dealt with one specific or a small number of business model components, such as the revenue [53] or the resource model [54], whereas a holistic approach remains an exception. An isolated investigation, however, contradicts the logic of business models as the components are interrelated and interdependent $[31,36]$.

The analysis of business models, in general, experienced a boom after the burst of the dot-com bubble in 2000. Scholars were searching for an explanation why a large number of companies had failed, whereas others remained successful $[36,55]$. It was observed that the same technology can result in significantly different economic output, depending on the way it is marketed by a business model [56]. The business model concept has thus played a central role in explaining a firm's performance and deriving success factors for a considerable time [35, 57]. The idea of factors, which are decisive for the success of a company, and that these can be determined, was first introduced in the 1960s by Daniel [58] and later mainly elaborated by Rockart [20]. According to Rockart [20], success factors are defined as "[...] the limited number of areas in which results, if they are satisfactory, will ensure successful competitive performance for the organization". Success factors are by definition applicable to all companies of a specific industry with similar objecttives and strategies $[19,20]$. In this paper, similar objectives and strategies are substituted by ecosystem roles as these possess specific business model characteristics. In literature, a basic distinction is often made with regard to the scope of application between generic success factors being valid for all kind of companies, and domain-specific, in this case cloud-specific, success factors [6]. Therefore, it would be error-prone to simply transfer success factors from adjacent research fields to the cloud ecosystem without prior examination [8].

The success factor research as a whole has developed into an independent research field, which, as the multitude of published studies shows, enjoys great popularity. However, it is also subject to criticism. According to Nicolai and Kieser [59], the success factor research has not been able to derive consistent, reliable and practically usable results. Furthermore, March and Sutton [60] argue that single success factors lose their value after having been identified and published. The reason for their assumption is that other firms would implement these success factors that once have distinguished high performers from low performers, whereby these success factors lose impact [60]. However, it has to be considered that companies have different starting situations, which are, according to the resource-based view [61], characterized by a specific set of limited resources. To realize all business models' success factors would hence not be possible from a resource point of view. Even supposing unlimited resources, it has to be kept in mind that a business model cannot be successful per se because it still has to be implemented in the form of business processes. A strong business model can be managed badly and therefore fail, whereas a 
weak business model may succeed due to great management and implementation skills [38]. This is according to Nenonen and Storbacka [27] also one reason why various business model designs can create solid financial results. To conclude, not every company which implements the valid success factors will inevitably become successful. The factors should be regarded rather as guidelines for orientation or recommended scope of actions for companies.

Specific research on success factors of cloud providers' business models is very limited [6-8]. Trenz et al. [7] focused on specific success factors referring to the relationship between providers and consumers in the end consumer market. Labes et al. [6] derived abstract success factors by relating publicly available information regarding predefined characteristics of business models to a firm's web visibility and profit. Concerning the SaaS provider's role, Floerecke [8] identified 27 success factors by conducting an exploratory multiple-case study. Besides, few isolated studies [13-16] exists in the SaaS field which, however, mainly focus on value propositions, while disregarding other business model components, or are solely literature-based, trying to summarize the fragmented scientific literature on SaaS or transferring success factors of related fields. The IaaS and PaaS providers' ecosystem roles have been widely neglected [17]. Only Floerecke and Lehner [17] proposed eight initial hypotheses on business model characteristics for small and medium-sized, locally operating IaaS providers for counteracting the increasing market consolidation and thus, the resulting dominance of the hyperscalers.

\section{RESEARCH DESIGN}

\subsection{Scope of Investigation}

The goal of this paper is to identify the success-driving business model characteristics of cloud providers adopting the ecosystem roles IaaS and/or PaaS provider. Figure 2 summarizes the specific scope of investigation:

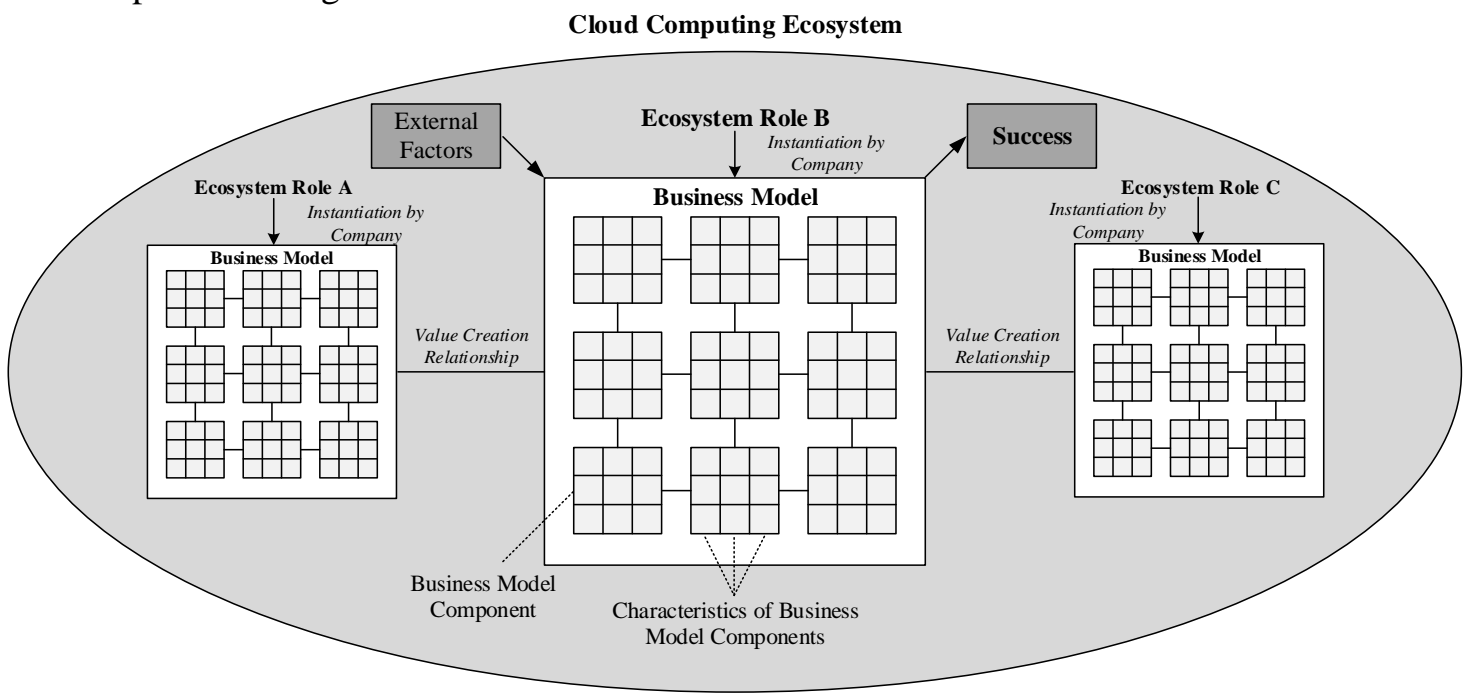

Figure 2. The Scope of Investigation

Figure 2 illustrates three randomly selected, directly connected roles $(\mathrm{A}, \mathrm{B}, \mathrm{C})$ within the cloud computing ecosystem. Each of these roles is occupied by a different cloud provider and implemented in the form of a separate business model. The companies are interconnected through their business models, more precisely, through value creation relationships. Each business model consists of several components, which can exhibit various characteristics. Of course, it is also possible that all of the three roles are occupied in common by only one company. The focus of this paper is placed on the investigation of the connection between individual characteristics within the different business model components with regard to a specific ecosystem role (IaaS and PaaS 
provider) and the role-specific economic success of the respective company. Multi-component patterns, this means, interactions between individual characteristics of various business model components are deliberately excluded, due to their complex and largely unexplored interconnections $[36,38,55]$ and the difficulty of collecting them reliably. Success is defined in accordance with the standard within the business model $[57,62]$ and the success factor $[19,20]$ research in financial terms, as return on investment (ROI). External factors, which imply a specific business model characteristic, are not subject of this study.

\subsection{Research Methodology}

To identify the success-driving business model characteristics of IaaS and PaaS providers thoroughly, a multiple-case study approach was selected. Yin [18] defines a case study as "[...] an empirical inquiry that investigates a contemporary phenomenon (the "case") in depth within its real-life context, especially when the boundaries between phenomenon and context may not be clearly evident". Case study research is suitable (i) to undertake research in widely unexplored areas, (ii) to answer "how", "why" and "what" questions and (iii) to learn about the state of the art and generate theories from practice $[18,63]$. All of these three factors apply to this study. Case studies have moreover particularly proven their effectiveness in identifying success factors (e.g., $[19,20])$. This case study followed the positivistic tradition. More precisely, an inductive approach was applied with the aim to reach predominantly exploratory conclusions [63, 64]. In line with that, the research process did not start with a specific hypothesis being tested. However, Eisenhardt [65] argues that a priori specification of constructs can be helpful for shaping the initial design of a study. Therefore, this research used existing concepts from the literature on cloud business models and success factors for initial framing. But "[...] no construct is guaranteed a place in the resultant theory" [65] because of the study's exploratory nature.

Expert interviews [66] were used as the central data collection instrument. This is the most common data collection technique in exploratory case studies [18]. Thereby, the success-driving business model characteristics were collected impartially, directly from the interviewed experts. This means, the opinions and convictions of the experts were decisive, which may not necessarily correspond to the actual approaches or the best-practices of the firms. Accordingly, it was made a conscious decision against the alternative, to collect objective company information and to statistically relate it to corporate key figures $[6,57]$. The main reason for this was the difficulty of gaining access to adequate data in both quantitative and qualitative terms. Moreover, studies (e.g., $[19,67,68])$ have found that the subjectively perceived and expressed success factors by particularly senior managers of successful firms widely comply with the factual and objective ones.

\subsection{Site Selection}

A multiple-case study was preferred over a single-case study approach as the intent was hypothesis and theory building and it allows cross-case analysis which yields more general research results and ensures internal validity [63,64]. The central units of analysis were cloud providers occupying the IaaS and/or the PaaS provider's ecosystem role. These were chosen for enabling literal (i.e., predicting similar results) and theoretical (i.e., predicting contrasting results) replication logics [18]: The respective provider had to fulfil at least one of the two ecosystem roles and had to be listed as a successful provider by a leading research and advisory company (e.g., Gartner and Forrester). In this way, it was ensured that the basic settings of the providers were the same (literal replication). For theoretical replication, providers with differing experience, size, geographic coverage, number of occupied ecosystem roles, target markets, served industries and assessment of the importance of cloud compared to on-premise were included. Due to the heterogeneity of the providers, it was possible to draw cross-case conclusions, as it replicated findings across all cases and helped to detect similar and contrasting results, which lead to gene- 
ralizable conclusions [18]. When a provider fulfilled more than one of the two roles successfully, the one with the higher ranking was preferred. This selection procedure resulted in seven cases for both the PaaS and the IaaS provider's role, respectively, represented by ten cloud providers. Four companies were thus investigated concerning both ecosystem roles. This allowed, in addition, exploring the success factors, which arise from the interaction of both roles in the same company. The number of cases is in line with Eisenhardt [65] who recommends four to ten cases to reach external validity. Due to the multi-layered architecture of cloud services and the associated close connection between IaaS, PaaS and SaaS providers, seven mainly SaaS providers were also selected using the same case selection approach. In this way, the valuable perspectives of customers and partners of IaaS and PaaS providers were included.

\subsection{Data Collection}

The study started with a systematic screening of the cloud providers' websites to gain in-depth background information regarding their strategies and their business models. This initial data collection was directly incorporated in the following expert interviews with one or two representatives from each company. The interview partners within the cloud providers were selected based on two criteria: the person should hold a managerial position and have responsibility for his organization's business models and strategies. By the predominant use of (senior) management positions as data source, the previously mentioned prerequisite for the direct collection of success factors within expert interviews was addressed. In total, fourteen interviews across ten IaaS/PaaS providers and seven across seven mainly SaaS providers were conducted. This paper presents the integrated results as the respective discussions were not limited to the chosen ecosystem role. Instead, the experts often drew on their experience gathered in other ecosystem roles (as provider, partner or client). This proved to be a major advantage because it significantly increased the data pool and thus, the generalizability of the findings.

All interviews were based on a pre-tested interview guide, encompassing open-ended questions. When designing the interview guide, it had to be considered, whether to ask with a framework, such as the Business Model Canvas [40], in mind or without a given set of business model components concerning success factors. In line with the study's exploratory character, the authors decided not to push the interviewees into a given framework. Instead, they should be given the chance to think and answer freely, independent of predefined components. This approach was also deemed more appropriate by both participants of the two pre-test sessions. One expert pointed out the unnecessary additional time effort which would be necessary to explain a specific business model framework with all its relationships in detail due to the inherent complexity in comparison to the expected minor added value with regard to the quality of the results.

The interview guide (available upon request from the authors) focused on collecting the successdriving business model characteristics from various perspectives. These perspectives were derived from the literature on success factors and business models: In line with Leidecker and Bruno [19], a firm-specific and an industry-level analysis were chosen. In addition, the dynamic character of business models and their characteristic of being a differentiation instrument [31,36] were covered. Finally, the risk perspective was included - several scholars (e.g., [69]) distinguish between positive and negative success factors - aiming to draw conclusions on the positive side. The interview guide had been iteratively revised based on discussion sessions among the authors and colleagues of the research department and two subsequent, already mentioned test interviews with two practitioners.

Table 1 provides the interview details for each case. The cloud providers are anonymized with Greek letters. The firm size has the two categories medium and large, according to the EU Recommendation 2003/361/EC. The third column depicts which of the three cloud ecosystem's core roles (IaaS, PaaS, SaaS) the respective provider occupies and which of these were included (in 
International Journal on Cloud Computing: Services and Architecture (IJCCSA) Vol. 8, No.6, December 2018

bold) in the case study. The last column represents the internal position of the interviewees. To ensure anonymity, the job titles were slightly adjusted where necessary. In summary, the 21 interviewed experts had between three and ten years' experience in the cloud computing field and held leading positions within their companies, with the exception of two consultants.

Table 1. Summary of Firms and Respondents in Data Collection

\begin{tabular}{|c|c|c|c|}
\hline Firm Label & Firm Size & Occupied and Selected Roles & Position of Interviewee \\
\hline Alpha & Large & SaaS, PaaS, laaS & Head of Marketing DACH \\
\hline Beta & Large & SaaS, laaS & Head of SaaS \\
\hline Gamma & Medium & Saas & Head of System Engineering \\
\hline Delta & Medium & Saas & Chief Financial Officer \\
\hline \multirow{2}{*}{ Epsilon } & \multirow{2}{*}{ Large } & \multirow{2}{*}{ SaaS, PaaS, laaS } & Senior Portfolio Manager Hybrid IT \\
\hline & & & Director Hybrid IT Central Europe \\
\hline Zeta & Medium & SaaS & Cloud Division Manager \\
\hline Eta & Large & SaaS, PaaS, laaS & Cloud Services Leader Europe \\
\hline \multirow{2}{*}{ Theta } & \multirow{2}{*}{ Large } & \multirow{2}{*}{ SaaS, PaaS, laaS } & Product Marketing Manager PaaS \\
\hline & & & Head of Partner Development \\
\hline lota & Large & SaaS & Senior Vice President \\
\hline Kappa & Medium & SaaS, PaaS, laaS & Principal IT Architect \\
\hline \multirow{2}{*}{ Lambda } & \multirow{2}{*}{ Large } & \multirow{2}{*}{ SaaS, PaaS, laaS } & Principal Sales Consultant \\
\hline & & & Middleware Partner Adoption Europe \\
\hline Mu & Medium & SaaS & Head of Product Management \\
\hline $\mathrm{Nu}$ & Large & laas & Vice President Sales DACH \\
\hline $\mathbf{X i}$ & Large & SaaS, PaaS & Vice President Product Management \\
\hline Omicron & Large & SaaS, PaaS & Portfoliomanager Platform Solutions \\
\hline $\mathbf{P i}$ & Large & laaS, PaaS & Senior Business Consultant \\
\hline \multirow{2}{*}{ Rho } & \multirow{2}{*}{ Large } & \multirow{2}{*}{ SaaS, PaaS, laaS } & Digital Consultant \\
\hline & & & Vice President Business Solutions \\
\hline
\end{tabular}

The 21 interviews took place from June to November 2017. The interview language was German. Ten interviews were done face-to-face, eleven by phone. Sturges and Hanrahan [70] have shown that there are no significant differences between a face-to-face and a telephone interview regarding the quality of the gathered data. The duration ranged from 30 to 100 minutes. The interview guide was deliberately not sent to the experts in advance as spontaneous responses were desired. Whenever appropriate, the laddering technique was applied, which follows a process of digging deeper by asking further questions [71]. Thus, the interviews were conducted as guided conversations rather than structured interviews. A possible lack of agreement between experts was not considered as problematic, but rather as an opportunity to explore and include differing perspectives. Once a disagreement appeared, it served as starting point to investigate the specific topic more in-depth in the remaining interviews. All interviews were recorded, transcribed, proof read and anonymized by the authors. The data collection was undertaken until the answers were repeated many times and no new aspects were added, so that further data collection would not 
have provided additional insights (theoretical saturation). This point was reached relatively quickly as the skilled and experienced cloud experts often also referred to other ecosystem roles.

\subsection{Data Analysis}

The data analysis was performed in two phases based on the recommendations of Corbin and Strauss [72] with the qualitative data analysis software MAXQDA: The first phase consisted of open coding - a line-by-line analysis of the transcribed data [72]. The components of the Business Model Canvas [40] served as the initial categories of the coding scheme. This framework was selected for the following reasons: First, it is comprehensive as it entails most of the other existing frameworks' components $[31,36]$. Second, it is widespread among both practitioners and scholars. Third, it was already used successfully in the cloud domain (e.g., [45]) and has thereby proved its suitability. Thus, its usage also improves the future comparability of the results. The derived codes were discussed iteratively among both authors until common agreement was reached. In the second phase, the codes were further consolidated using axial coding [72]. This resulted in 40 main codes, representing the success-driving business model characteristics of IaaS and/or PaaS providers. The whole data analysis was an iterative process of (re-)coding data, splitting and combining categories, and generating new or dropping existing categories.

An aspect was classified as success-driving, if it was expressed as such by various experts, or in case of a single notion, when convincing arguments were provided, or if it emerged as an important aspect during analysis of an individual case or the cross-case comparisons. In line with Corbin and Strauss [72], no attempt was made to statistically evaluate the importance of the results. Theoretical relevance of the concepts was established by their repeated presence or notable absence. In this paper, only business model characteristics being directly related to the IaaS and PaaS providers' ecosystem roles are included. Every success factor was further classified by the authors as generic, similar to other domains, or cloud-specific. In order to structure the success factors and in accordance with the data analysis process, they were sorted into the Business Model Canvas. When a success factor referred to several business model components, it was assigned to that being considered primarily affected.

\section{SuCCESS-DRIVING BUSiness Model CharaCteristics}

The identified success-driving business model characteristics of the cloud ecosystem roles IaaS and PaaS provider are presented and described separately according to the components of the Business Model Canvas. Due to the close connection of the components customer relationships and channels, both are thereafter combined into one component. The classification whether the respective success factor is generic ([g]), similar to other domains, or cloud-specific ([s]) and its assignment to both ecosystem roles is noted in brackets after the designation of each factor.

\subsection{Value Propositions}

(1) Offering and Supporting Multi-Cloud Management (IaaS) [s]. The growing importance of multi-cloud management was stressed. Its underlying idea is to offer one's own IaaS service and additionally act as a broker for other providers. This is because of the simultaneous use of IaaS services from various providers by the majority of the medium-sized and large companies: (i) several SaaS services need to be deployed on the IaaS/PaaS platform of the specific provider, (ii) employees sometimes order IaaS services without prior approval by the IT department and (iii) clients try to actively avoid vendor lock-in. Concerning (iii), several clients prefer to have even three or more different IaaS providers simultaneously, regardless of the actual active use.

(2) Offering a Wide Range of Integrated PaaS Elements (PaaS) [s]. As the clients' needs vary, developing a broad PaaS portfolio with a large number of different, but integrated services is crucial. The smaller the components are the more flexible they can be combined, but this makes it 
less transparent and more complex for clients to pick and connect the desired services. It has proved to cluster PaaS services according to their use case. Specifically, it is important to support services from a variety of third-party providers and open source elements. The portfolio has be continuously expanded and kept up-to-date. Presently, it is recommended to cover container services, big data, machine learning, blockchain, artificial intelligence and Internet of Things.

(3) Offering Integration PaaS Services (PaaS) [s]. There is a strong demand for integration PaaS services. This means that the PaaS platform acts as a central integration point where offered SaaS services and also on-premise solutions can be integrated by customers. For this purpose, the provider has to offer a variety of predefined connectors. Such a service is particularly promising for PaaS providers that have a large client base for SaaS. Thus, the SaaS portfolio acts as a driver for the PaaS platform.

(4) Offering Managed Services (IaaS) [s]. Standard IaaS services consist of basic virtual compute, storage and network resources. Besides that, there is a growing market for managed services. Managed services entail an extension comprising elements such as update, monitoring or backup services. Especially among firms which formerly were traditional IT outsourcing clients a high demand can be found. These companies are used to pass the responsibility for the complete IT operations on to the provider. In contrast, start-ups often prefer basic IaaS services. IaaS providers can also instruct partner firms to offer managed services.

(5) Guaranteeing a High Availability (IaaS, PaaS) [g]. It is absolutely imperative to ensure a high availability of the offered cloud services. However, negative incidents in the past prove how challenging this undertaking is. A long-lasting service failure comes along with a significant loss of customer confidence, leading to adverse effects on the provider's economic situation. Hence, the specification and compliance of the service level agreements is very relevant.

(6) Achieving a High User Experience (IaaS, PaaS) [g]. The handling of cloud services must be self-explanatory and simple, to enable immediate use. Traditional concepts which have been utilized within large on-premise software systems are less suitable. Customers desire an uncomplicated user experience that they are accustomed to in their private environment. This has also the advantage to reduce training course expenditures for customers' employees.

(7) Covering Hybrid Cloud Scenarios (IaaS) [s]. Many firms' IT landscapes are still characterized by a large proportion of legacy on-premise systems. Nevertheless, several firms have already begun to obtain additional private and public clouds. Hence, a considerable market arises to combine the old and the new worlds of IT by hybrid cloud scenarios. Thus, it is crucial that the public cloud complements and expands the private cloud, so that clients can shift workloads between the various systems easily. It is a serious mistake to offer an isolated public cloud.

(8) Offering Private Clouds (IaaS, PaaS) [s]. The demand for private clouds is currently considerably higher than for public clouds. Many clients consider private clouds as an interim solution on their way to public cloud. Private clouds are preferred due to data protection, security, availability, regulation and compliance reasons. In addition, a private cloud allows a substantially higher degree of customization which clients often demand. Ultimately, it is mandatory to include private clouds in the portfolio.

(9) Offering Extensive Transition Services From On-Premise to Cloud (IaaS) [s]. Mediumsized and large enterprises that have accumulated lots of on-premise infrastructure face a challenge when deciding to move specific parts of their IT to the cloud. Only in rare cases, the migration of the systems can be managed without external help. Consequently, there is a strong demand for support and transition services. During this transitional period, extensive consulting and customizing services are necessary to get a firm cloud-ready. Supplying transition services has an additional strategic aspect as a provider can directly win customers for his IaaS services.

(10) Offering a Broad and Integrated Cloud Portfolio (IaaS, PaaS) [g]. Offering a multitude of cloud services across all service layers and deployment models is promising. When creating a 
broad portfolio, one should avoid many isolated components and instead, concentrate on a high degree of integration. First, this leads to a high attractiveness of a provider as clients feel more prepared for the future. Second, clients always look for an answer to their specific requirements. By means of a broad portfolio a provider can better respond to that demand. Third, when a provider has full control over the service provision, he can offer higher security and quality.

(11) Providing Extensive Client Support (IaaS, PaaS) [g]. Providing extensive support for the cloud service lifecycle is essential. This includes services related to the selection, implementation and operation of cloud services. Providers should closely work with clients as they want to call for help anytime a problem occurs. The support process has to be integrated so that clients have to talk to only one person to identify and solve problems. Extensive support is particularly seen as a way for providers with low innovation strength to differentiate from competitors.

(12) Offering Customer-Specific Service Adaption (IaaS, PaaS) [g]. Cloud services are characterized by a high degree of standardization. However, it is essential to preserve a certain degree of flexibility. Some customers have additional requirements that cannot be entirely met by standard services. Customization is associated with additional costs, but a willingness to pay can be expected if an added value is guaranteed.

\subsection{Key Resources}

(1) Locating Data Centres in the Target Country (IaaS) [s]. It is crucial for success, especially in the German IaaS market, that cloud providers have their data centres located in the respective country. In this respect, customers are highly sensitive due to performance and latency, legal, cultural and emotional reasons.

(2) Operating a Worldwide Network of Interconnected Data Centres (IaaS) [s]. It is not enough to operate a single data centre. To prevent loss of data, additional backup and recovery data centres are needed. To address geographic risks, these have to be located in considerable distance from one another. Between the data centres, a high-speed data connection with low latency has to be established. It not only increases the failure security, but also leads to global coverage. Thus, clients from (almost) all over the world are able to use the IaaS service with convenient performance.

(3) Building and Operating Innovative, Cost-Efficient Data Centres (IaaS) [s]. The experts stressed the significant differences between the designs of data centres of the various IaaS providers. The hyperscalers' data centres are characterized by innovativeness and engineering performance. In order to establish oneself as a new player, considerable knowledge and experience are a prerequisite, whereas trial and error is not an option. The main goals are to reduce energy costs and to operate data centres in the most cost-efficient way, while guaranteeing stability, scalability and performance.

(4) Possessing the Leading Certificates (IaaS, PaaS) [s]. Cloud providers have to obtain the relevant certificates. The importance of certificates varies in relation to client size and type of cloud service: whereas small firms may partly consider certificates as dispensable, they are vital in an enterprise context. Only applications working solely with insensitive data commonly do not demand certificates. As the certification process is time-consuming and expensive, small providers can hardly compete.

(5) Using an Open Source Platform (IaaS) [s]. The majority of the hyperscalers developed and utilizes a proprietary IaaS platform. Using an open source IaaS platform, however, has the following advantages: First, it helps to avoid the vendor lock-in. By using an open source IaaS platform, clients can easier switch from one provider to another, if both providers support the open standard. Second, providers can save the royalty payments. These savings can be passed on to the customers. Third, providers that are actively involved in the open source community benefit from 
the accumulated know-how and best-practices. Fourth, open standards are a prerequisite to realize cloud native SaaS applications in the form of microservices.

(6) Having a Multitude of Highly Qualified Employees (PaaS, IaaS) [g]. The experts emphasized the importance of having a multitude of highly qualified employees. This particularly includes software developers who implement the cloud portfolio. Considering PaaS, a provider requires persons with a deep understanding of software development. Besides that, employees with technical know-how are needed to support clients in case of uncertainties and problems over the cloud service lifecycle.

(7) Owning a Large (Pre-Cloud) Customer Base (IaaS, PaaS) [g]. A large customer base that a provider established before the emergence of cloud computing is regarded a high-valued resource as it provides easy and fast access to potential cloud customers. This is firstly the case as longstanding and satisfied clients have confidence in the provider's qualities. Secondly, the clients know the provider's support processes and do not want to contact another provider when problems occur. Thirdly, clients are used to the specific applications, so they are commonly reluctant to change the provider.

(8) Building Up Domain/Industry Expertise (PaaS) [g]. Despite the importance of technical aspects of cloud service implementation, it is necessary for PaaS providers to accumulate a broad domain knowledge and industry expertise in the fields they operate in. If providers are not able to build this up in-house, they must rely on partners that possess such knowledge. This is a prerequisite to ensure that a PaaS service, e.g., an industrial Internet of Things platform, delivers added value for customers.

\subsection{Key Activities}

(1) Using Agile Development Models (PaaS) [s]. Traditional, sequential development models, such as the Waterfall Model [73], are not appropriate for cloud services. Instead, agile methods like Scrum [73] are substantial to realize short innovation cycles. Development speed is a decisive factor: new services or additional features have to be delivered continuously and fast on the platform in order to improve the portfolio. In this regard, DevOps plays an important role, meaning that there has to be a close link between development and operation of any cloud service.

(2) Conducting Research and Development (IaaS, PaaS) [g]. A high-level research and development is vital in the rapidly changing cloud ecosystem. The risk of lagging behind the technological development is high, which is why many cloud providers are not only carrying out inhouse research and development, but also acquire smaller providers to increase their service portfolio and knowledge.

\subsection{Partner Network}

(1) Building a Partner Ecosystem (IaaS, PaaS) [s]. A thriving partner ecosystem serves as a sales and marketing channel: cloud services are highly scalable, but it is impractical to interact personally with each customer as the sheer volume of sales staff for this cannot be supported. Hence, partners can act as resellers. Important partners are also firms undertaking consulting and implementation projects. Overall, closer client proximity, further geographic coverage and client segments that do not fall into the provider's primary scope can be achieved. Moreover, a provider cannot perform all services outside his core business himself. These services, which are elementary to enable optimum use of a cloud service, include, e.g., training and support.

(2) Waiving of Partners in the Core Services (IaaS) [s]. IaaS providers should strictly avoid having partners in their core services. It is common practice to have a data centre operator being responsible for the energy supply and the cooling facilities. But, e.g., an IaaS provider advertising his data centres' location in Europe cannot have a sub-provider outside the continent who occasionally stores parts of the data. This is seen as a reason for the failure of some small provi- 
ders. Overall, it is difficult to fulfil the service level agreements in terms of, e.g., security and availability, as the question of responsibility arises. It is also prohibited by several certificates.

(3) Bringing External Developer Firms and Customers Together on the Platform (PaaS) [s]. External developer firms can build SaaS applications or refine and expand existing SaaS services on the basis of the provider's PaaS platform. These new SaaS services should be offered on the PaaS provider's market place. Third-party software can also be offered, even if it has not been built with the platform. Clients are thus able to order the applications over a uniform platform without contacting each vendor independently. A PaaS platform should therefore act as an active enabler for business models of other firms and bring clients and developers together.

\subsection{Customer Segments}

(1) Focusing on Ambitious Medium-Sized and Large Companies (IaaS, PaaS) [s]. It is recommended to focus on a specific customer segment. The more sophisticated medium-sized and large companies are considered as the most valuable one: First, providers can further sell on premise solutions and offer hybrid clouds on the basis of the clients' legacy IT systems. Second, many small firms lag behind in technological advances as well as business trends. Third, larger firms have more financial resources at their disposal to pay for cloud services.

(2) Offering the Possibility for Firms of All Sizes to Become a Client (IaaS, PaaS) [g]. Although it is primarily important to concentrate on a specific segment, providers should also cater other possible customers. Small firms should obtain the opportunity to order a cloud service via self-service and pay with credit card. Another option is to distribute special cloud services for small companies through partners. To reach larger firms, a provider has to offer personal support. The overall target should be to reach as many clients as possible because a user of a small firm per se generates the same amount of turnover as one of a large firm.

\subsection{Customer Relationships and Channels}

(1) Offering Personal Instead of Self-Service Sale (IaaS, PaaS) [g]. The acceptance of selfservice purchase decreases from IaaS toward SaaS. A reason for this is that SaaS and PaaS services often have to be integrated in the clients' processes and IT landscapes. While small firms intensively use the self-service option, larger firms commonly insist on personal contact to the provider combined with individual contract negotiations. In general, the self-service variant only makes sense when offering standardized cloud services. Practice shows that several clients have difficulties in using self-service sale, mainly due to a lack of skills. To conclude, depending on the cloud service and the target group, it is crucial to put emphasis on personal sale.

(2) Initial Explaining of the Cloud Concept (IaaS, PaaS) [s]. Many potential cloud customers have considerable doubts whether they should move into the cloud. The central concern is data security. Oftentimes the source of these doubts is a lack of knowledge. The experts stated that firms are commonly not aware of the differences between IT outsourcing and cloud computing. It is crucial to address these fears and unknowingness at the beginning of the customer relationship by explaining the conditions of cloud computing in detail. This is a valuable contribution to establish trust.

(3) Establishing and Maintaining Close Contact to the Developer Community (PaaS) [s]. For $\mathrm{PaaS}$ providers it is essential to establish and maintain a close alliance with the developer community. Otherwise, a PaaS provider will not receive consideration. However, the experts reported that it is very challenging to gain a foothold within this community. It is necessary to disclose any information on the website about the internal PaaS platform operation and functionalities. In addition, it has become evident to operate a central exchange platform, such as a forum or a blog, where developers can share information on problems and best-practices. Close contact to developers is also a valuable source for feedback as a basis for future improvements. 
(4) Conducting Marketing Activities (IaaS, PaaS) [g]. To stand out from the huge number of providers, marketing is of great significance. In addition, due to the short contractual periods in the cloud field, the customer royalty is decoupled. Further, the decision on the client's side in favour of a specific provider is often not based on performance features. Instead, the provider's image is a decisive factor. Marketing is expected to gain further in importance as the decisionmaking power moves increasingly from IT towards business departments. Promising marketing channels are presentations at conferences, publication of articles, cooperation with universities, use of social media and build-up of communities. Moreover, the value of reference customers for newly developed cloud services was emphasized.

(5) Establishing a Cloud-Specific Incentive System for the Sales Staff (IaaS, PaaS) [s]. The incentive system for the sales division for on-premise solutions is not directly transferable to the cloud. Traditionally, it has been possible for a salesperson to make deals at regular intervals with one client, whereby he received his commissions regularly. In the case of subscription-based pricing models, clients usually pay a usage fee for a longer period of time. After the first sale, no salesperson is needed any more. Moreover, by selling a cloud service the salesperson takes away his future upselling options as clients receive an all-inclusive deal. In the case of usage-based pricing models, it is known only ex post, which amount of resources the client really used. As all this has drastic effects on bonus payments, it is crucial to develop a cloud-specific internal incentive system to promote motivation within the sales division.

\subsection{Revenue Streams}

(1) Keeping Up With the Market Prices - Price as Main Sales Argument (IaaS) [s]. Basic public IaaS services have become a commodity. Therefore, the price has turned into the most important decision criterion for clients. Providers that are unable to keep up with the market prices will gradually be forced out of the IaaS market, if they do not develop strong distinguishing features. It is necessary to pass the increasing savings of economies of scale and technological progress on to the clients.

(2) Providing Transparency and Simplicity in Usage-Based Pricing Models (IaaS, PaaS) [s]. The most important characteristic of usage-based pricing models is transparency and simplicity. The actual implementation becomes a secondary consideration. This means, the pricing model should be based on a small number of components, which enable detailed consumption monitoring, so that clients have a chance to clearly see which elements form the pricing model. Clients want to know how they can actively influence their costs incurred through their usage behaviour, estimate in advance, what expenses will roughly occur and compare prices for similar services of various providers.

(3) Offering Fixed Prices for Permanent Public Cloud Service Usage (IaaS, PaaS) [s]. Several clients prefer fixed prices for a public cloud when they already have a precise knowledge of the amount of resources they will probably need for a specific period of time. Moreover, many firms are not capable to use usage-based pricing models due to budget limitations, as they need to apply for money internally in advance. Furthermore, several decision makers simply continue to favour traditional fix prices which they are used to. But as customers have to enter a long-term contract, they often expect price discounts in return.

(4) Implementing a Layer-Overarching Pricing Model (IaaS, PaaS) [s]. Providers often face the problem that clients only use a small number of their IaaS/PaaS services. A major reason is that clients usually have to subscribe to each service separately in direct contact with the provider followed by a new contract negotiation. This required effort may be discouraging for clients. A promising solution is to implement a cloud layer-overarching credit point system: clients buy credits (an artificial currency) in advance, whereby they are able to purchase any cloud service from the portfolio directly. Clients can thus use the whole cloud portfolio with a high flexibility. 
(5) Supporting of "Bring Your Own License" (IaaS, PaaS) [s]. To attract new clients, move long-term on-premise clients into the cloud or move up clients to a higher cloud layer, it is crucial to allow bringing already purchased licenses of on-premise software for a corresponding cloud service. This is challenging in case the license stems from a third-party provider: An onpremise license has a specific calculation basis, usually the client's CPU cores. But due to hardware virtualization, it is difficult to ensure that the service solely runs on the allowed number of CPUs in the cloud.

(6) Offering Flexibility within the Pricing Model (IaaS, PaaS) [s]. Besides offering predefined pricing models, an open attitude towards individual requests from customers concerning the design of the pricing model can have a strong effect on customer satisfaction. This means a pricing model should, in certain cases, take the characteristics of the business model and the situation of different customers into account.

\subsection{Cost Structure}

(1) Reducing Electrical Consumption (IaaS) [s]. Electricity has been identified as the main cost driver of IaaS providers. The most electricity is needed for the cooling system. Hence, IaaS providers have to pay particular attention to reducing electricity consumption. One method is to relocate some data centres to geographic areas with a cooler climate, e.g., Northern Europe. Another option is to invest in more energy-efficient hardware.

(2) Achieving Economies of Scale (IaaS) [s]. The main goal of providers of public IaaS is to obtain economies of scale. Naturally, small providers are at disadvantage. An IaaS provider has to reach a certain size to reduce the costs per service unit. Otherwise, he will not be able to keep up with the dropping prices for basic IaaS services.

\section{DISCUSSION}

\subsection{Critical Discussion of the Success-Driving Business Model Characteristics}

This study identified 40 success-driving business model characteristics of the cloud ecosystem roles IaaS and/or PaaS provider. Most of them refer to value propositions, key resources, customer relationships, channels and revenue streams, whereas key activities, partner networks, customer segments and cost structure were hardly addressed by the experts. 22 of the identified business model characteristics are applicable to both of the investigated ecosystem roles. Hence, it can be concluded that these are, at least partly, also relevant for further roles of the cloud ecosystem. This assumption is supported by the findings of Floerecke [8], whose identified SaaS-specific success factors correspond to a large degree with the results regarding role-overarching success factors in this paper. On the other hand, this study revealed a variety of role-specific factors. This reflects the fact that the roles of the cloud ecosystem are characterized by a high level of heterogeneity, which makes a role-specific investigation and thus, an ecosystemic perspective imperative. Overall, both generic and cloud-specific factors could be determined. It can therefore be concluded that in the cloud ecosystem exist generic as well as cloud-specific, subdivided into roleoverarching and role-specific, success factors. However, apart from a few exceptions in the SaaS field, so far cloud business models have mostly been studied comprehensively, independently from the distinctly different cloud ecosystem roles.

Upon closer examination of the identified success-driving business model characteristics, it becomes evident that the preferences of clients do not entirely correspond with or even partly contradict the scientific, conceptual definition of cloud computing. This includes, firstly, addressing client-specific requests regarding pricing models and value propositions. In literature (e.g., [21, 24]), cloud services are commonly defined by a high level of standardization. Secondly, ordering cloud services via self-service - one of the five key characteristics of cloud computing - is for a variety of reasons hardly accepted by customers, in particular among medium-sized and large 
companies. Personal contact is clearly preferred. These two aspects, however, contradict the providers' central goal of achieving economies of scale. Thirdly, although IaaS and PaaS are mostly associated with usage-based pricing models (e.g., [21, 23]), there is a substantial demand for fixed prices in business practice. Furthermore, a clear majority of the considered cloud providers does, contrary to the recommendation of scholars (e.g., [36, 39]), not undergo a systematic, phaseoriented process in the business model development and innovation process as such. It is rather conducted within occasional workshops, in which new ideas concerning the business models are collected and subsequently released for implementation.

The identified success factors differ considerably from those of Labes et al. [6]. Only a few are mentioned in both studies. This includes 4.1. $(7,8,9,11), 4.2$. $(6,8)$ and 4.7. (3). One main reason for this discrepancy is the different research design: Labes et al. [6] determined the success factors by screening the websites of cloud providers based on a predefined set of hypothetical business model characteristics. Thereby, the permissible value set for potential success factors had been restricted in advance. In contrast, the success factors were collected impartially, directly from the experts in the field in this study. In this light, the differing results are no surprise.

\subsection{Discussion of Current and Future Developments within the Cloud Ecosystem}

Beside the success-driving business model characteristics, this study brought widely unexpected aspects regarding the current situation and possible future developments within the IaaS and PaaS markets to light: Firstly, a major challenge concerning the IaaS business model is associated with the growing diffusion of cloud native applications. The key reason for this evolution is the increasing awareness among cloud providers that traditional on-premise software is, per se, not cloudready. It cannot meet the corresponding requirements (e.g., costs, performance and scalability). As a result, providers have to transform or rebuild the application - recommended as modular microservices by the interviewees. Microservices are characterized by the ability to autonomously manage their need of IaaS resources for operating a specific task. In addition, they can be run directly with various IaaS providers and combined and customized according to the customer's specific requirements. However, particularly traditional large-scale applications, which are commonly structured as a monolithic block, require considerable effort to make them cloud-ready. A promising strategy is to develop each new application as a cloud native version because it must only be slightly adapted to be sold as on-premise. As a consequence, the main concern of IaaS providers will probably no longer be to solely offer a virtual server as an IaaS service because it is becoming less common that clients order a predefined amount of compute, storage and network resources. Hence, the common IaaS business model is expected to change drastically in the midterm future. If this happens indeed, parts of the success-driving business model characteristics identified in this study will likely be affected.

Moreover, PaaS is currently gaining significant popularity among customers as they can immediately use fully-fledged services. A growing number of providers have namely included readyto-use PaaS services in their portfolio, which customers formerly had to develop on their own upon an IaaS service. In addition, as PaaS often contains elements of machine learning and artificial intelligence, it can provide considerable added value to customers. Until now, the PaaS providers focus on slightly different areas, which make comparison difficult. Nonetheless, the experts are firmly convinced that the battle for market shares will be fought particularly within the PaaS provider's role over the next years, making it the most important one for cloud providers. It can already be observed that more and more providers are taking steps towards PaaS both coming from IaaS and SaaS. Established PaaS providers are putting much effort into expanding and improving their portfolio. However, as this paper has shown, the requirements for successful PaaS value propositions are high (see section 4.1. (2)). 
Basic IaaS services have become a commodity. Providers that are unable to keep up with the market prices will hence gradually be forced out of the IaaS market, if they do not develop any strong distinguishing characteristics, e.g., extensive support and consulting or customer-specific adaptions. A growing market consolidation, particularly among small and medium-sized, locally operating IaaS providers can already be observed. If this continues, the IaaS market is, according to the experts, expected to become subject of governmental regulations in future, similar to other utility markets, such as the market for electrical energy. Otherwise, the remaining hyperscalers could use their dominance for arbitrary pricing. The increasing loss of importance of basic IaaS services with regard to differentiation is apparent in the fact that the leading providers do not structure their cloud portfolio according to IaaS and PaaS anymore. Instead, they bundle both into one integrated offer and sell it under one label, whereby IaaS is only the vehicle to supply PaaS. Offering basic IaaS services solely is no longer a profitable business. Several experts even consider this approach a business model error. A provider should adopt both the IaaS and the PaaS provider's role. This assumption is also supported by a current study of Gartner [12]. The roles' underlying business models offer synergy effects. Supplying PaaS on the basis of another provider's IaaS service is, inter alia, due to differing standards, out of the question.

\section{CONCLUSiON}

In order to identify the business model characteristics which contribute to the success of cloud providers adopting the ecosystem roles IaaS and/or PaaS provider, an exploratory multiple-case study was conducted. 21 expert interviews with representatives from 17 cloud providers served as central instrument of data collection. The result is a catalogue of 40 success-driving business model characteristics. Overall, it could be shown that in the cloud ecosystem exist generic as well as cloud-specific, subdivided into role-overarching and role-specific, success factors. This finding underpins the fact that the roles of the cloud ecosystem are characterized by a high level of heterogeneity, making a role-specific investigation of business models indispensable. Among the identified success factors some are particularly surprising as they are not consistent with the scientific, conceptual definition of cloud computing. This includes addressing client-specific requests, e.g., regarding pricing models and value propositions, providing personal instead of selfservice sale and offering fixed prices for IaaS and PaaS usage. Beyond that, this study brought unexpected aspects regarding the current situation and possible future developments in the IaaS and PaaS markets to light: (1) the major foreseeable changes within the IaaS business model as a result of the growing diffusion of cloud native applications, (2) the forecasted evolution of the PaaS provider's role toward the most decisive ecosystem role and (3) the shrinking perspectives for pure providers of basic IaaS services as those have become a commodity.

This paper is the first to address the research gap regarding the success-driving business model characteristics of IaaS and PaaS providers. The paper's further contribution for researchers lies in the promising starting and reference point for future analysis of business models of various cloud ecosystem roles. The comprehensive Passau Cloud Computing Ecosystem Model (PaCE Model) [9], which was developed through a systematic analysis and synthesis of the previously published cloud computing ecosystem models, provides an appropriate basis for this undertaking. The PaCE Model comprises 26 roles for market actors, which are grouped into five categories - (1) client, (2) vendor, (3) hybrid role, (4) support and (5) environment - and entails the basic service flows between the roles [9]. Therefore, this study contributes to the literature on cloud ecosystems and business models by linking both previously widely unrelated research streams. Within the cloud ecosystem, a business model is defined as a detailed specification of how ecosystem roles are realized by individual companies. As a whole, this paper is the first, which has taken a perspective shift from an overall towards a selective, role-specific and thus ecosystemic perspective on business models in the cloud computing domain. 
Nevertheless, the study's findings are equally valuable for practitioners: Established IaaS and/or PaaS providers obtain a reference framework to compare, rethink and, if deemed necessary, innovate their present business models. Companies that are planning to offer IaaS and/or PaaS in future gain valuable insights that should directly feed into their business model design process. In order to enable and facilitate the feasibility of the derived business model characteristics by practitioners, they were deliberately defined on a low abstraction level and described with a relatively high level of detail. However, at this point, it has to be emphasized again that a business model cannot be successful per se as it still has to be implemented in the form of business processes. This means that not every company which implements the business model characteristics identified in this paper will inevitably become successful. The factors should be considered as guidelines for orientation or recommended scope of actions for cloud providers.

The authors are aware that this study has some limitations: First, the collected data and thus the findings are only as reliable as the given answers of the interviewees. A final evidence for the validity of the identified success-driving business model characteristics cannot be provided. Second, it was neglected that companies fulfilling the same ecosystem role can be, due to various strategies, sizes or lifecycle phases, affected by a specific success factor to a different extent. A third limitation is the unequal distribution of success factors within the business model components. This could reflect the actual distribution, but it could also be caused by a limited knowledge of the interviewees of the wide scope of the business model construct. Researchers are recommended to apply a given business model framework for asking regarding the success factors in future. Fourth, the geographic scope of the case sites was restricted to Germany. But as the majority of cases consisted of international providers, this influence is considered as relatively low. Fifth, mainly large providers were part of this study. However, this could not be avoided as small providers only play a minor role in the fields of IaaS and PaaS. Nevertheless, future studies could specifically focus on small and medium-sized IaaS and PaaS providers. Sixth, to broaden the view, it would have been promising to include providers that have not been successful or that already had to withdraw from the cloud ecosystem. Individual examples of the last-mentioned category were requested for an interview, but none of them agreed to participate. In addition, besides the integration of SaaS experts, it also would have been useful to interview representatives from consulting firms in order to benefit from their in-depth experience concerning the whole cloud ecosystem.

Despite the valuable results, a substantial need for further research remains: First, as not all business model characteristics are of equal importance, their relevance has to be ranked. This could be carried out, for instance, within a survey or a Delphi study. Second, it should be examined to which extent the success factors, which are visible from outside, are currently covered by the various IaaS and PaaS providers. In this context, it is of great interest to compare the coverage between successful and less successful cloud providers. Third, this study has primarily taken a snapshot of success factors. As success factors can, however, change over time, they have to be reassessed in future regarding their ongoing validity. Fourth, the focus was placed on the isolated effects of individual business model characteristics. But as a business model is a system of interrelated components, the combined effects of specific characteristics have to be investigated in future. In this regard, possible incompatibilities have to be explored. Due to the selected research design, the individual interviewees did not necessarily cover a multitude of the business model components and thus, their mutual interactions. These additional insights would greatly increase the value of the results for practitioners. Fifth, it is necessary to investigate business models of further roles that are part of the PaCE Model. Cloud brokers, such as aggregators, integrators and market place operators, are recommended to be addressed next due to their major importance for the ecosystem's functioning. In this regard, it should be assessed to what extent the successdriving business model characteristics of IaaS and PaaS providers are transferable. Finally, to 
support practitioners it is mandatory to deal intensively with the development process of business models as such because research in this area is still in its infancy.

It will be interesting to watch the evolution of the IaaS and PaaS segments, in particular: Will there be fundamental changes of the IaaS business models in the light of the growing diffusion of cloud native applications? To what extent will the forecasted market consolidation among IaaS providers take actually place? Which providers will win the announced battle for market shares in the PaaS field - mostly those coming from SaaS or those from IaaS? Will PaaS also become a commodity over the next years?

\section{REFERENCES}

[1] Clohessy, T., Acton, T., Morgan, L., and Conboy, K. (2016), "The Times They Are A-Chaning For ICT Service Provision: A Cloud Computing Business Model Perspective", presented at the European Conference on Information Systems, Istanbul, Turkey.

[2] DaSilva, C. M., Trkman, P., Desouza, K., and Lindič, J. (2013), "Disruptive Technologies: A Business Model Perspective on Cloud Computing", Technology Analysis \& Strategic Management, Vol. 25, No. 10, pp. 1161-1173.

[3] Henkes, A., Heuer, F., Vogt, A., Heinhaus, W., Giering, O., and Landrock, H. (2016), "Cloud Vendor Benchmark 2016: Cloud Computing Anbieter im Vergleich", Experton Group, Ismaning, Germany.

[4] Smith, D., Leong, L., and Bala, R. (2018), "Magic Quadrant for Cloud Infrastructure as a Service, Worldwide", Gartner, Stamford, USA.

[5] Costello, K. and van der Meulen, R. (2018), "Gartner Says Worldwide IaaS Public Cloud Services Market Grew 29.5 Percent in 2017", Gartner, Stamford, USA.

[6] Labes, S., Hanner, N., and Zarnekow, R. (2017), "Successfull Business Model Types of Cloud Providers", Business \& Information Systems Engineering, Vol. 59, No. 4, pp. 223-233.

[7] Trenz, M., Huntgeburth, J., and Veit, D. (2017), "How to Succeed with Cloud Services?", Business \& Information Systems Engineering, pp. 1-14.

[8] Floerecke, S. (2018), "Success Factors of SaaS Providers' Business Models - An Exploratory Multiple-Case Study", presented at the International Conference on Exploring Service Science, Karlsruhe, Germany.

[9] Floerecke, S. and Lehner, F. (2016), "Cloud Computing Ecosystem Model: Refinement and Evaluation", presented at the European Conference on Information Systems, Istanbul, Turkey.

[10] Leimeister, S., Böhm, M., Riedl, C., and Krcmar, H. (2010), "The Business Perspective of Cloud Computing: Actors, Roles and Value Networks", presented at the European Conference on Information Systems, Pretoria, South Africa.

[11] Floerecke, S. and Lehner, F. (2015), "A revised Model of the Cloud Computing Ecosystem", presented at the International Conference on Economics of Grids, Clouds, Systems, and Services, Cluj-Napoca, Romania.

[12] Costello, K. and Hippold, S. (2018), "Gartner Forecasts Worldwide Public Cloud Revenue to Grow 17.3 Percent in 2019", Gartner, Stamford, USA.

[13] Ernst, C. P. H. and Rothlauf, F. (2012), "Potenzielle Erfolgsfaktoren von SaaS-Unternehmen", presented at the Multikonferenz Wirtschaftsinformatik, Braunschweig, Germany.

[14] Walther, S., Plank, A., Eymann, T., Singh, N., and Phadke, G. (2012), "Success Factors and Value Propositions of Software as a Service Providers - A Literature Review and Classification", presented at the American Conference on Information Systems, Seattle, USA.

[15] Wieneke, A., Walther, S., Eichin, R., and Eymann, T. (2013), "Erfolgsfaktoren von On-DemandEnterprise-Systemen aus der Sicht des Anbieters - Eine explorative Studie", presented at the International Conference on Wirtschaftsinformatik, Leipzig, Germany.

[16] Biggeleben, M., Kolbe, H., Schäfermeyer, M., and Vranesic, H. (2009), "Prüfkriterien für Geschäftsmodelle im Kontext von Software as a Service", presented at the Internationale Tagung Wirtschaftsinformatik, Wien, Austria.

[17] Floerecke, S. and Lehner, F. (2018), "Business Model Characteristics for Local IaaS Providers for Counteracting the Dominance of the Hyperscalers", presented at the International Conference on Economics of Grids, Clouds, Systems, and Services, Pisa, Italy. 
International Journal on Cloud Computing: Services and Architecture (IJCCSA) Vol. 8, No.6, December 2018

[18] Yin, R. K. (2014), Case Study Research: Design and Methods, 5. ed., Thousand Oaks: Sage Publications.

[19] Leidecker, J. K. and Bruno, A. V. (1984), "Identifying and Using Critical Success Factors", Long Range Planning, Vol. 17, No. 1, pp. 23-32.

[20] Rockart, J. F. (1979), "Chief Executives Define their Own Data Needs", Harvard Business Review, Vol. 57, No. 2, pp. 81-93.

[21] Marston, S., Li, Z., Bandyopadhyay, S., Zhang, J., and Ghalsasi, A. (2011), "Cloud Computing The Business Perspective", Decision Support Systems, Vol. 51, No. 1, pp. 176-189.

[22] Böhm, M., Koleva, G., Leimeister, S., Riedl, C., and Krcmar, H. (2010), "Towards a Generic Value Network for Cloud Computing", presented at the International Workshop on Economics of Grids, Clouds, Systems, and Services, Ischia, Italy.

[23] Mell, P. and Grance, T. (2011), "The NIST Definition of Cloud Computing", NIST, Gaithersburg, USA.

[24] Zhang, Q., Cheng, L., and Boutaba, R. (2010), "Cloud Computing: State-of-the-Art and Research Challenges", Journal of Internet Services and Applications, Vol. 1, No. 1, pp. 7-18.

[25] Adner, R. (2017), "Ecosystem as Structure: An Actionable Construct for Strategy", Journal of Management, Vol. 43, No. 1, pp. 39-58.

[26] Benedict, M. (2018), "Modelling Ecosystems in Information Systems - A Typology Approach", presented at the Multikonferenz Wirtschaftsinformatik, Lüneburg, Germany.

[27] Nenonen, S. and Storbacka, K. (2010), "Business Model Design: Conceptualizing Networked Value Co-Creation", International Journal of Quality and Service Sciences, Vol. 2, No. 1, pp. 4359.

[28] Myllykoski, J. and Ahokangas, P. (2012), "Transformation Toward a Cloud Business Model", Communications of the Cloud Software, Vol. 2, No. 1, pp. 1-11.

[29] Morris, M., Schindehutte, M., and Allen, J. (2005), "The Entrepreneur's Business Model: Toward a Unified Perspective", Journal of Business Research, Vol. 58, No. 6, pp. 726-735.

[30] Iivari, M. M., Ahokangas, P., Komi, M., Tihinen, M., and Valtanen, K. (2016), "Toward Ecosystemic Business Models in the Context of Industrial Internet", Journal of Business Models, Vol. 4, No. 2, pp. 42-59.

[31] Zott, C., Amit, R., and Massa, L. (2011), "The Business Model: Recent Developments and Future Research", Journal of Management, Vol. 37, No. 4, pp. 1019-1042.

[32] Tian, C. H., Ray, B. K., Lee, J., Cao, R., and Ding, W. (2008), "BEAM: A Framework for Business Ecosystem Analysis and Modeling", IBM Systems Journal, Vol. 47, No. 1, pp. 101-114.

[33] Labes, S., Erek, K., and Zarnekow, R. (2013), "Common Patterns of Cloud Business Models", presented at the Americas Conference on Information Systems, Chicago, Illionis.

[34] Aversa, P., Haefliger, S., and Reza, D. G. (2017), "Building a Winning Business Model Portfolio", MIT Sloan Management Review, Vol. 58, No. 4, pp. 49-54.

[35] Veit, D., Clemens, E., Benlian, A., Buxmann, P., Hess, T., Spann, M., et al. (2014), "Business Models - An Information Systems Research Agenda", Business \& Information Systems Engineering, Vol. 56, No. 1, pp. 55-64.

[36] Wirtz, B., Pistoia, A., Ullrich, S., and Göttel, V. (2016), "Business Models: Origin, Development and Future Research Perspectives", Long Range Planning, Vol. 49, No. 1, pp. 36-54.

[37] Amit, R. and Zott, C. (2001), "Value Creation in E-Business", Strategic Management Journal, Vol. 22, No. 6-7, pp. 493-520.

[38] Osterwalder, A., Pigneur, Y., and Tucci, C. L. (2005), "Clarifying Business Models: Origins, Present, and Future of the Concept", Communications of the Association for Information Systems, Vol. 16, No. 1, pp. 1-25.

[39] Foss, N. J. and Saebi, T. (2017), "Fifteen Years of Research on Business Model Innovation: How Far Have We Come, and Where Should We Go?", Journal of Management, Vol. 43, No. 1, pp. 200-227.

[40] Osterwalder, A. and Pigneur, Y. (2010), Business Model Generation: A Handbook for Visionaries, Game Changers, and Challengers, 1. ed., New Jersey: John Wiley \& Sons.

[41] Giessmann, A. and Stanoevska-Slabeva, K. (2012), "Business Models of Platform as a Service (PaaS) Providers: Current State and Future Directions", Journal of Information Technology Theory and Application, Vol. 13, No. 4, pp. 31-55.

[42] Giessmann, A. and Legner, C. (2016), "Designing Business Models for Cloud Platforms", Information Systems Journal, Vol. 26, No. 5, pp. 551-579. 
International Journal on Cloud Computing: Services and Architecture (IJCCSA) Vol. 8, No.6, December 2018

[43] Naous, D., Schwarz, J., and Legner, C. (2017), "Analytics As A Service: Cloud Computing and the Transformation of Business Analytics Business Models and Ecosystems", presented at the European Conference on Information Systems, Guimarães, Portugal.

[44] Kiesow, A. and Thomas, O. (2017), "Entwurf eines Cloud-basierten Geschäftsmodells für die kontinuierliche Prüfung", presented at the Internationalen Tagung Wirtschaftsinformatik, St. Gallen, Switzerland.

[45] Boillat, T. and Legner, C. (2013), "From On-Premise Software to Cloud Services: The Impact of Cloud Computing on Enterprise Software Vendors' Business Models", Journal of Theoretical and Applied Electronic Commerce Research, Vol. 8, No. 3, pp. 39-58.

[46] Khanagha, S., Volberda, H., and Oshri, I. (2014), "Business Model Renewal and Ambidexterity: Structural Alteration and Strategy Formation Process During Transition to a Cloud Business Model", R\&D Management, Vol. 44, No. 3, pp. 322-340.

[47] Kranz, J. J., Hanelt, A., and Kolbe, L. M. (2016), "Understanding the Influence of Absorptive Capacity and Ambidexterity on the Process of Business Model Change - The Case of On-Premise and Cloud-Computing Software", Information Systems Journal, Vol. 26, No. 5, pp. 477-517.

[48] Clohessy, T., Acton, T., and Morgan, L. (2018), "Contemporary Digital Business Model Decision Making: A Cloud Computing Supply-Side Perspective", International Journal of Networking and Virtual Organisations, Vol. 19, No. 1, pp. 1-20.

[49] Ebel, P., Bretschneider, U., and Leimeister, J. M. (2016), "Leveraging Virtual Business Model Innovation: A Framework for Designing Business Model Development Tools", Information Systems Journal, Vol. 26, No. 5, pp. 519-550.

[50] Habjan, K. B. and Pucihar, A. (2017), "The Importance of Business Model Factors for Cloud Computing Adoption: Role of Previous Experiences", Organizacija, Vol. 50, No. 3, pp. 255-272.

[51] Hentschel, R., Leyh, C., and Petznick, A. (2018), "Current Cloud Challenges in Germany: The Perspective of Cloud Service Providers", Journal of Cloud Computing: Advances, Systems and Applications, Vol. 7, No. 1, pp. 1-12.

[52] Labes, S., Erek, K., and Zarnekow, R. (2013), "Literaturübersicht von Geschäftsmodellen in der Cloud", presented at the Internationale Tagung Wirtschaftsinformatik, Leipzig, Germany.

[53] Al-Roomi, M., Al-Ebrahim, S., Buqrais, S., and Ahmad, I. (2013), "Cloud Computing Pricing Models: A Survey", International Journal of Grid and Distributed Computing, Vol. 6, No. 5, pp. 93-106.

[54] Herzfeldt, A., Floerecke, S., Ertl, C., and Krcmar, H. (2018), "The Role of Value Facilitation Regarding Cloud Service Provider Profitability in the Cloud Ecosystem," in Multidisciplinary Approaches to Service-Oriented Engineering, M. Khosrow-Pour, Ed., 1. ed., Hershey: IGI Global, pp. 121-142.

[55] Burkhart, T., Krumeich, J., Werth, D., and Loos, P. (2011), "Analyzing the Business Model Concept - A Comprehensive Classification of Literature", presented at the International Conference on Information Systems, Shanghai, China.

[56] Chesbrough, H. and Rosenbloom, R. S. (2002), "The Role of the Business Model in Capturing Value from Innovation: Evidence from Xerox Corporation's Technology Spin-off Companies", Industrial and Corporate Change, Vol. 11, No. 3, pp. 529-555.

[57] Lambert, S. C. and Davidson, R. A. (2013), "Applications of the Business Model in Studies of Enterprise Success, Innovation and Classification: An Analysis of Empirical Research from 1996 to 2010", European Management Journal, Vol. 31, No. 6, pp. 668-681.

[58] Daniel, D. R. (1961), "Management Information Crisis", Harvard Business Review, Vol. 39, No. 5, pp. 111-121.

[59] Nicolai, A. and Kieser, A. (2002), "Trotz eklatanter Erfolglosigkeit: die Erfolgsfaktorenforschung weiter auf Erfolgskurs", Die Betriebswirtschaft, Vol. 62, No. 6, pp. 579-596.

[60] March, J. G. and Sutton, R. I. (1997), "Crossroads - Organizational Performance as a Dependent Variable", Organization Science, Vol. 8, No. 6, pp. 698-706.

[61] Bharadwaj, A. S. (2000), "A Resource-Based Perspective on Information Technology Capability and Firm Performance: An Empirical Investigation", Management Information Systems Quarterly, Vol. 24, No. 1, pp. 169-196.

[62] Zott, C. and Amit, R. (2007), "Business Model Design and the Performance of Entrepreneurial Firms", Organization Science, Vol. 18, No. 2, pp. 181-199.

[63] Benbasat, I., Goldstein, D. K., and Mead, M. (1987), "The Case Research Strategy in Studies of Information Systems", Management Information Systems Quarterly, Vol. 11, No. 3, pp. 369-386. 
International Journal on Cloud Computing: Services and Architecture (IJCCSA) Vol. 8, No.6, December 2018

[64] Dubé, L. and Paré, G. (2003), "Rigor in Information Systems Positivist Case Research: Current Practices, Trends, and Recommendations", Management Information Systems Quarterly, Vol. 27, No. 4, pp. 597-636.

[65] Eisenhardt, K. M. (1989), "Building Theories from Case Study Research", Academy of Management Review, Vol. 14, No. 4, pp. 532-550.

[66] Myers, M. D. and Newman, M. (2007), "The Qualitative Interview in IS Research: Examining the Craft", Information and Organization, Vol. 17, No. 1, pp. 2-26.

[67] Grunert, K. G. and Sørensen, E. (1996), "Perceived and Actual Key Success Factors: A Study of the Yoghurt Market in Denmark", Aarhus School of Business, Aarhus, Denmark.

[68] Hoffmann, W. H. and Schlosser, R. (2001), "Success Factors of Strategic Alliances in Small and Medium-Sized Enterprises - An Empirical Survey", Long Range Planning, Vol. 34, No. 3, pp. 357-381.

[69] Fritz, W. (1993), "Die empirische Erfolgsfaktorenforschung und ihr Beitrag zum Marketing: Eine Bestandsaufnahme", Technische Universität Braunschweig, Braunschweig, Germany.

[70] Sturges, J. E. and Hanrahan, K. J. (2004), "Comparing Telephone and Face-to-Face Qualitative Interviewing: A Research Note", Qualitative Research, Vol. 4, No. 1, pp. 107-118.

[71] Corbridge, C., Rugg, G., Major, N. P., Shadbolt, N. R., and Burton, A. M. (1994), "Laddering: Technique and Tool Use in Knowledge Acquisition", Knowledge Acquisition, Vol. 6, No. 3, pp. 315-341.

[72] Corbin, J. and Strauss, A. (2008), Basics of Qualitative Research: Techniques and Procedures for Developing Grounded Theory, 1. ed., Thousand Oaks: SAGE Publications.

[73] Ruparelia, N. B. (2010), "Software Development Lifecycle Models", ACM SIGSOFT Software Engineering Notes, Vol. 35, No. 3, pp. 8-13.

\section{AUTHORS}

Sebastian Floerecke graduated with both a Bachelor's and a Master's degree in Information Systems at Technical University of Munich (Germany) in 2011 and 2014, respectively. During his studies, he worked as a student assistant at the Chair for Information Systems (Prof. Dr. Helmut Krcmar) in the research fields of product service systems and enterprise architecture management. In late 2014, Sebastian Floerecke joined the Chair of Information Systems (Information and IT Service Management) of Uni-

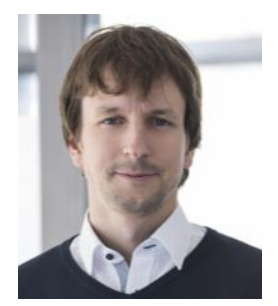
versity of Passau held by Prof. Dr. Franz Lehner, where he is currently research associate and doctoral candidate. His academic work focuses on cloud computing business models and ecosystems.

Franz Lehner has been assistant at the Institute for Organizational Research at the University of Linz (JKU), Austria, since 1986. Before this he gathered experience in the field of EDP as head of the educational centre at a software house and as an independent consultant. From 1992 to 1994, he was professor for business administration and information management at the WHU Koblenz School of Corporate Management (Germany), followed by a professorship for Information Systems at the University of Regensburg.

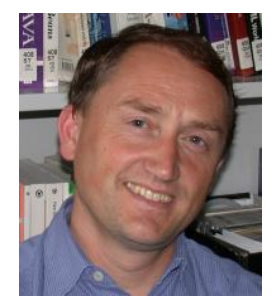
Since 2004 he holds the chair of Information Systems specializing on Information and IT Service Management at the University of Passau. Research activities are focused on knowledge and information management, cloud computing and development of mobile applications. 